\title{
Light propagation in the gravitational field of moving bodies by means of Lorentz transformation I. Mass monopoles moving with constant velocities
}

\author{
S. A. Klioner ${ }^{\star}$ \\ Lohrmann Observatory, Dresden Technical University, Mommsenstr. 13, 01062 Dresden, Germany \\ Received 28 January 2003 / Accepted 31 March 2003

\begin{abstract}
We show how to derive the equations of light propagation in the gravitational field of uniformly moving mass monopoles without formulating and integrating the differential equations of light propagation in that field. The well-known equations of light propagation in the gravitational field of a motionless mass monopole are combined with a suitable Lorentz transformation. The possibility of generalizing this technique for the more complicated case of uniformly moving body of arbitrary multipole structure is discussed.
\end{abstract}

Key words. astrometry - reference systems - relativity - gravitational lensing

\section{Introduction}

Within the next decade the accuracy of space-based astrometric positional observations is expected to attain 1 microarcsecond ( $\mu$ as). This technical progress is expected to be achieved due to a number of space astrometry projects (e.g., GAIA - ESA 2000; Perryman et al. 2001; Bienayme \& Turon 2002 - and SIM - Shao 1998 - approved by ESA and NASA). Modeling of the observed data with such an accuracy is a highly non-trivial task that requires the use of general relativity. The whole reduction scheme should be consequently formulated within the framework of general relativity. Recently, a practical relativistic model for positional observations of microarcsecond accuracy performed from space has been formulated by Klioner (2003). In particular, many subtle relativistic effects in light propagation should be accounted for to attain the goal accuracy of $1 \mu$ as. One of the most intricate points of the whole relativistic model is to compute the effects of the translational motion of gravitating bodies on the light propagation.

This problem was treated probably for the first time by Hellings (1986). He recommended to use the standard postNewtonian formulas for the light propagation in the gravitational field of a motionless body and to substitute in those formulas the position of each gravitating body at the moment of closest approach of that body and the photon. The next step has been done by Klioner (1989) where the problem was solved completely for bodies moving with a constant velocity in the first post-Newtonian approximation. The effects of accelerations of the bodies have been further treated by

^ e-mail:klioner@rcs.urz.tu-dresden.de
Klioner \& Kopeikin (1992) where it was shown that if the coordinates and velocities of the bodies are computed at the moments of closest approach of the corresponding body and the photon, the residual terms of the solution are in some sense minimized. The complete solution of the problem for arbitrarily moving bodies in the first post-Minkowskian approximation was found by Kopeikin \& Schäfer (1999) who succeeded in integrating analytically the post-Minkowskian equations of light propagation in the field of arbitrarily moving mass monopoles (see Appendix $\mathrm{C}$ for an explicit form of the Kopeikin-Schäfer solution). These authors used the representation of the metric tensor through the Lienard-Wiechert potentials with retarded arguments. The Kopeikin-Schäfer scheme has been generalized by Kopeikin \& Mashhoon (2002) to the case of arbitrarily moving bodies possessing mass monopoles and spin dipoles.

Extensive numerical simulations of light propagation in the time-dependent gravitational field of the solar system have been recently done by Klioner \& Peip (2003). That publication contains also detailed information of all the approaches mentioned above. The aim of the numerical simulations was to check the practical accuracy of various approximate analytical solutions for the light propagation in the field of moving bodies and verify the practical recommendations formulated by Klioner (2003).

Up to now all theoretical results concerning light propagation in the field of moving bodies concerned moving mass monopoles (i.e. "point masses"). It is well known, however, that for GAIA and other space missions not only the mass monopoles, but also the gravitational fields produced by higher multipoles (especially, by mass quadrupoles) are important (Klioner 2003). This paper is the first one in a series of papers 
where the light propagation in the gravitational field of moving bodies with full multipole structure will be investigated. The aim of this paper is to show that the Lorentz transformation can be used to derive the laws of light propagation in the gravitational field of a system of uniformly moving bodies in the first post-Minkowskian (or post-Newtonian) approximation of general relativity.

Let us clarify here that the post-Minkowskian approximation scheme deals with expansions in powers of the gravitational constant $G$. The first post-Minkowskian approximation implies that all terms of order $O\left(G^{2}\right)$ are neglected. The postNewtonian approximation scheme operates with expansions in powers of $c^{-1}$. In the first post-Newtonian approximation terms of order $O\left(c^{-4}\right)$ are neglected in the equations of light propagation. One can prove that in the case of light propagation the formulas of the first post-Newtonian approximation are linear in $G$ and, therefore, contained in those of the first post-Minkowskian approximation.

It is well known that the differential equations of light propagation in both the post-Newtonian and post-Minkowskian approximations are linear with respect to the non-Galilean components of the metric tensor $h_{\alpha \beta}$ :

$g_{\alpha \beta}=\eta_{\alpha \beta}+h_{\alpha \beta}$

with

$\eta_{\alpha \beta}=\operatorname{diag}(-1,+1,+1,+1)$

i.e. linear with respect to the gravitational constant $G$. It is also clear that in this approximation the gravitational potentials $h_{\alpha \beta}$ can be written as a sum of the contributions coming from each body of a material system. Therefore, the gravitational influences of each body on the light propagation can be considered independently of each other.

Let us first assume that each body moves with a constant velocity relative to a reference system $\left(t, x^{i}\right)$. Then, in order to derive the influence of a rectilinearly and uniformly moving body $A$ on the light propagation one can simply introduce a reference system $\left(T, X^{a}\right)_{A}$ where the body is at rest. Reference systems $\left(T, X^{a}\right)_{A}$ and $\left(t, x^{i}\right)$ can be related to each other by the usual Lorentz transformation. In reference system $\left(T, X^{a}\right)_{A}$ the laws of light propagation are very well known: these are the laws of light propagation in the gravitational field of a motionless body (i.e. mass monopole (Schwarzschild) field or gravitational field with a given multipole structure). Then applying the Lorentz transformation to those known laws of light propagation one restores the more complicated equations of light propagation in the gravitational field of a uniformly moving body $A$. This very transparent scheme allows one to re-derive and better understand the formulas for the light propagation in the field of moving bodies found recently by Kopeikin \& Schäfer (1999) and Kopeikin \& Mashhoon (2002), and also generalize these solutions for the case of uniformly moving bodies with full multipole structure.

\section{General considerations: Uniform motion}

\subsection{Lorentz transformation}

Since each body $A$ in the system can be considered in the same way, the subscript $A$ and the corresponding sums will be omitted below. Lorentz transformations between the reference system $\left(t, x^{i}\right)$ and the reference system $\left(T, X^{a}\right)$ where the body is at rest can be written in the form (throughout the paper repeated indices imply Einsteinian summation rule irrespective of their positions)

$c t=\Lambda_{0}^{0} c T+\Lambda_{a}^{0} X^{a}$,

$x^{i}=\Lambda_{0}^{i} c T+\Lambda_{a}^{i} X^{a}$,

where

$\Lambda_{0}^{0}=\gamma$,

$\Lambda_{a}^{0}=k^{a} \gamma$

$\Lambda_{0}^{i}=k^{i} \gamma$,

$\Lambda_{a}^{i}=\delta^{i a}+\frac{\gamma^{2}}{1+\gamma} k^{i} k^{a}$

$\gamma=(1-\boldsymbol{k} \cdot \boldsymbol{k})^{-1 / 2}$,

$\boldsymbol{k}=\boldsymbol{v} / c$.

Here and below a dot between two coordinate 3-vectors denotes a scalar product with respect to the Euclidean metric, for example, $\boldsymbol{k} \cdot \boldsymbol{k}=k^{a} k^{a}$. Velocity $\boldsymbol{v}$ is the parameter of the transformation that will be discussed below. The inverse transformations read

$c T=\bar{\Lambda}_{0}^{0} c t+\bar{\Lambda}_{i}^{0} x^{i}$

$X^{a}=\bar{\Lambda}_{0}^{a} c t+\bar{\Lambda}_{i}^{a} x^{i}$

where

$\bar{\Lambda}_{0}^{0}=\gamma$,

$\bar{\Lambda}_{i}^{0}=-k^{i} \gamma$

$\bar{\Lambda}_{0}^{a}=-k^{a} \gamma$

$\bar{\Lambda}_{i}^{a}=\delta^{i a}+\frac{\gamma^{2}}{1+\gamma} k^{i} k^{a}$

\subsection{Position of the body}

Let $X_{A}^{a}=$ const be the coordinates of the body in the reference system $\left(T, X^{a}\right)$. Transforming events $\left(T, X_{A}^{a}\right)$ into the reference system $\left(t, x^{i}\right)$ yields

$\boldsymbol{x}_{A}(t)=\boldsymbol{x}_{A}\left(t_{A}\right)+\boldsymbol{v}_{A}\left(t-t_{A}\right)$,

where

$v_{A}^{i}=c \Lambda_{0}^{i} / \Lambda_{0}^{0}=v^{i}$

and $t_{A}$ can be chosen arbitrarily. These formulas mean that the body that is at rest in the reference system $\left(T, X^{a}\right)$ moves with a constant velocity $\boldsymbol{v}_{A}=\boldsymbol{v}$ in the reference system $\left(t, x^{i}\right)$. This velocity serves as the parameter of the transformations (3)-(6). 


\subsection{Unperturbed trajectory of the photon}

The unperturbed trajectory of the photon $X_{\mathrm{p}}^{a}(T)$ in the reference system $\left(T, X^{a}\right)$ is a straight line

$\boldsymbol{X}_{\mathrm{p}}(T)=\boldsymbol{X}_{\mathrm{p} 0}+c \boldsymbol{\Sigma}\left(T-T_{0}\right)$,

where $\boldsymbol{X}_{\mathrm{p} 0}=$ const is the position of the photon for $T=T_{0}$ and $\boldsymbol{\Sigma}$ is the unit direction of the light propagation $(\boldsymbol{\Sigma} \cdot \boldsymbol{\Sigma}=$ $1)$. Transforming the events $\left(T, X_{\mathrm{p}}^{a}(T)\right)$ into the reference system $\left(t, x^{i}\right)$ one gets

$x_{\mathrm{p}}(t)=x_{\mathrm{p} 0}+c \sigma\left(t-t_{0}\right)$,

where

$c t_{0}=\Lambda_{0}^{0} c T_{0}+\Lambda_{a}^{0} X_{\mathrm{p} 0}^{a}$,

$x_{\mathrm{p} 0}^{i}=\Lambda_{0}^{i} c T_{0}+\Lambda_{a}^{i} X_{\mathrm{p} 0}^{a}$,

and

$\sigma^{i}=\frac{\Lambda_{0}^{i}+\Lambda_{a}^{i} \Sigma^{a}}{\Lambda_{0}^{0}+\Lambda_{a}^{0} \Sigma^{a}}$.

Therefore, one has $\sigma \cdot \sigma=1$. The inverse transformation of $\sigma$ into $\Sigma$ has exactly the same form as (12) but with the corresponding components on the inverse Lorentz transformation:

$\Sigma^{a}=\frac{\bar{\Lambda}_{0}^{a}+\bar{\Lambda}_{i}^{a} \sigma^{i}}{\bar{\Lambda}_{0}^{0}+\bar{\Lambda}_{i}^{0} \sigma^{i}}$

\subsection{First-order perturbations of the light ray}

Let us write the perturbed trajectory of the photon $X_{\mathrm{p}}^{a}(T)$ in the reference system $\left(T, X^{a}\right)$ as

$\boldsymbol{X}_{\mathrm{p}}(T)=\boldsymbol{X}_{\mathrm{p} 0}+c \boldsymbol{\Sigma}\left(T-T_{0}\right)+\Delta \boldsymbol{X}_{\mathrm{p}}(T)$,

with

$\Delta \boldsymbol{X}_{\mathrm{p}}\left(T_{0}\right)=0$,

$\lim _{T \rightarrow-\infty} \frac{\mathrm{d}}{\mathrm{d} T} \Delta \boldsymbol{X}_{\mathrm{p}}(T)=0$.

Transforming the events $\left(T, X_{\mathrm{p}}^{a}(T)\right)$ as defined by (14) one gets

$\boldsymbol{x}_{\mathrm{p}}(t)=\boldsymbol{x}_{\mathrm{p} 0}+c \boldsymbol{\sigma}\left(t-t_{0}\right)+\Delta \boldsymbol{x}_{\mathrm{p}}(t)$,

with

$\Delta \boldsymbol{x}_{\mathrm{p}}\left(t_{0}\right)=0$,

$\lim _{t \rightarrow-\infty} \frac{\mathrm{d}}{\mathrm{d} t} \Delta x_{\mathrm{p}}(t)=0$,

and $\boldsymbol{\sigma}$ and $\boldsymbol{\Sigma}$ are related to each other by the same Eqs. (12) and (13) as in the unperturbed case. The light path perturbation in the reference system $\left(t, x^{i}\right)$ reads

$\Delta x_{\mathrm{p}}^{i}(t)=\left(\Lambda_{a}^{i}-\sigma^{i} \Lambda_{a}^{0}\right) \Delta X_{\mathrm{p}}^{a}(T)$.

It is useful also to derive the corresponding transformation formulas for the coordinate velocity of the photon. In the reference system $\left(T, X^{a}\right)$ one gets

$\boldsymbol{V}_{\mathrm{p}}(T) \equiv \frac{\mathrm{d}}{\mathrm{d} T} \boldsymbol{X}_{\mathrm{p}}(T)=c \boldsymbol{\Sigma}+\Delta \boldsymbol{V}_{\mathrm{p}}(T)$, where

$\Delta \boldsymbol{V}_{\mathrm{p}}(T)=\frac{\mathrm{d}}{\mathrm{d} T} \Delta \boldsymbol{X}_{\mathrm{p}}(T)$.

From (16) one has

$\boldsymbol{v}_{\mathrm{p}}(t) \equiv \frac{\mathrm{d}}{\mathrm{d} t} \boldsymbol{x}_{\mathrm{p}}(t)=c \boldsymbol{\sigma}+\Delta \boldsymbol{v}_{\mathrm{p}}(t)$

with

$\Delta v_{\mathrm{p}}(t)=\frac{\mathrm{d}}{\mathrm{d} t} \Delta x_{\mathrm{p}}(t)$.

The Lorentz transformation gives

$v_{\mathrm{p}}^{i}=c \frac{\Lambda_{0}^{i}+\Lambda_{a}^{i} V_{\mathrm{p}}^{a} / c}{\Lambda_{0}^{0}+\Lambda_{a}^{0} V_{\mathrm{p}}^{a} / c}$.

Taking $t \rightarrow-\infty$ in (23) one restores the relation between $\sigma$ and $\boldsymbol{\Sigma}$ given by (12). Therefore, the relation between $\Delta v_{\mathrm{p}}(t)$ and $\Delta \boldsymbol{V}_{\mathrm{p}}(T)$ reads

$$
\begin{aligned}
\Delta v_{\mathrm{p}}^{i}(t) & =\frac{1}{\Lambda_{0}^{0}+\Lambda_{b}^{0} V_{\mathrm{p}}^{b}(T) / c}\left(\Lambda_{a}^{i}-\sigma^{i} \Lambda_{a}^{0}\right) \Delta V_{\mathrm{p}}^{a}(T) \\
& =\left(\bar{\Lambda}_{0}^{0}+\bar{\Lambda}_{j}^{0} v_{\mathrm{p}}^{j}(t) / c\right)\left(\Lambda_{a}^{i}-\sigma^{i} \Lambda_{a}^{0}\right) \Delta V_{\mathrm{p}}^{a}(T),
\end{aligned}
$$

which can be simplified to

$$
\Delta v_{\mathrm{p}}^{i}(t)=\left(\bar{\Lambda}_{0}^{0}+\bar{\Lambda}_{j}^{0} \sigma^{j}\right)\left(\Lambda_{a}^{i}-\sigma^{i} \Lambda_{a}^{0}\right) \Delta V_{\mathrm{p}}^{a}(T)+O\left(G^{2}\right)
$$

in the first post-Minkowskian approximation. The same equation can be derived directly from (18).

Now substituting some specific expressions for $\Delta X_{\mathrm{p}}(T)$ and/or $\Delta \boldsymbol{V}_{\mathrm{p}}(T)$ (e.g. those for the Schwarzschild field or for the gravitational field with some multipole structure) one can derive the corresponding perturbations $\Delta x_{\mathrm{p}}(t)$ and $\Delta v_{\mathrm{p}}(t)$ without integration of any additional differential equations of light propagation in the reference system where the body moves.

\section{Mass monopoles in uniform motion}

The solution for the light propagation in the gravitational field of a motionless mass monopole in the first post-Minkowskian approximation is very well known (the post-Newtonian and post-Minkowskian solutions coincide in this case) and was published e.g. by Will (1993). In our notations one has

$\Delta \boldsymbol{X}_{\mathrm{p}}(T)=-\frac{2 G M}{c^{2}}\left(\boldsymbol{F}(T)-\boldsymbol{F}\left(T_{0}\right)\right)+O\left(G^{2}\right)$

$\boldsymbol{F}(T)=\frac{\boldsymbol{D}}{|\boldsymbol{R}|-\boldsymbol{\Sigma} \cdot \boldsymbol{R}}-\boldsymbol{\Sigma} \log (|\boldsymbol{R}|-\boldsymbol{\Sigma} \cdot \boldsymbol{R})$,

$\frac{1}{c} \Delta \boldsymbol{V}_{\mathrm{p}}(T)=-\frac{2 G M}{c^{2}}\left(\frac{\boldsymbol{D}}{|\boldsymbol{R}|(|\boldsymbol{R}|-\boldsymbol{\Sigma} \cdot \boldsymbol{R})}+\frac{\boldsymbol{\Sigma}}{|\boldsymbol{R}|}\right)+O\left(G^{2}\right)$,

$\boldsymbol{D}=\boldsymbol{\Sigma} \times(\boldsymbol{R} \times \boldsymbol{\Sigma})$,

$\boldsymbol{R}=\boldsymbol{X}_{\mathrm{p} 0}+c \boldsymbol{\Sigma}\left(T-T_{0}\right)-\boldsymbol{X}_{A}$,

where $\boldsymbol{X}_{A}$ is the constant position of the body. Note that $\boldsymbol{D}$ is a time-independent vector. 
Applying the Lorentz transformations (3)-(6) to each element of (26)-(30) (the most important parts of this transformation are given in Appendix A) and using (18) and (25) one gets

$$
\begin{aligned}
\Delta \boldsymbol{x}_{\mathrm{p}}(t)= & -\frac{2 G M}{c^{2}}\left(\boldsymbol{f}(t)-\boldsymbol{f}\left(t_{0}\right)\right)+O\left(G^{2}\right), \\
\boldsymbol{f}(t)=\frac{\boldsymbol{r}-\frac{1}{\theta}(\boldsymbol{\sigma} \cdot \boldsymbol{r})(\boldsymbol{\sigma}-\boldsymbol{k})}{\left(\rho-\frac{1}{\gamma \theta} \boldsymbol{\sigma} \cdot \boldsymbol{r}+\gamma \boldsymbol{k} \cdot \boldsymbol{r}\right)} & \quad-(\boldsymbol{\sigma}-\boldsymbol{k}) \log \left(\rho-\frac{1}{\gamma \theta} \boldsymbol{\sigma} \cdot \boldsymbol{r}+\gamma \boldsymbol{k} \cdot \boldsymbol{r}\right), \\
\frac{1}{c} \Delta \boldsymbol{v}_{\mathrm{p}}(t)= & -\frac{2 G M}{c^{2}} \gamma \theta\left[\frac{\boldsymbol{r}-\frac{1}{\theta}(\boldsymbol{\sigma} \cdot \boldsymbol{r})(\boldsymbol{\sigma}-\boldsymbol{k})}{\rho\left(\rho-\frac{1}{\gamma \theta} \boldsymbol{\sigma} \cdot \boldsymbol{r}+\gamma \boldsymbol{k} \cdot \boldsymbol{r}\right)}\right. \\
& \left.\quad+\frac{\gamma(\boldsymbol{\sigma}-\boldsymbol{k})}{\rho}\right]+O\left(G^{2}\right), \\
\rho=\left(|\boldsymbol{r}|^{2}\right. & \left.+\gamma^{2}(\boldsymbol{k} \cdot \boldsymbol{r})^{2}\right)^{1 / 2},
\end{aligned}
$$

where $\boldsymbol{r}$ and $\theta$ are defined by (A.5) and (A.2), respectively. Neglecting in (31)-(34) all the terms of order $O\left(k^{2}\right)$ (that is retaining all the terms at most linear relative to the velocity of the body) we immediately restore the corresponding formulas derived by Klioner $(1989,1991)$ and Klioner \& Kopeikin (1992) for the case of uniformly moving bodies in the first postNewtonian approximation.

Equations (31)-(33) can be drastically simplified by using the positions $x_{A}^{i}\left(t_{*}\right)$ of the gravitating body at the retarded moments of time $t_{*}$ and $t_{0 *}$ corresponding to the moments $t$ and $t_{0}$ as described in Appendix B:

$$
\begin{aligned}
& \Delta \boldsymbol{x}_{\mathrm{p}}(t)=-\frac{2 G M}{c^{2}}\left(\boldsymbol{f}_{*}(t)-\boldsymbol{f}_{*}\left(t_{0}\right)\right)+O\left(G^{2}\right), \\
& \boldsymbol{f}_{*}(t)=\gamma\left(\theta \frac{\boldsymbol{d}_{*}}{p_{*}}-(\boldsymbol{\sigma}-\boldsymbol{k}) \log p_{*}\right), \\
& \frac{1}{c} \Delta \boldsymbol{v}_{\mathrm{p}}(t)=-\frac{2 G M}{c^{2}} \frac{\gamma \theta}{\left|\boldsymbol{r}_{*}\right|-\boldsymbol{k} \cdot \boldsymbol{r}_{*}} \\
& \times\left(\theta \frac{\boldsymbol{d}_{*}}{p_{*}}+(2-\theta) \boldsymbol{\sigma}-2 \boldsymbol{k}\right)+O\left(G^{2}\right),
\end{aligned}
$$$$
p_{*}=\left|\boldsymbol{r}_{*}\right|-\boldsymbol{\sigma} \cdot \boldsymbol{r}_{*},
$$

where $\boldsymbol{d}_{*}$ is defined by (B.7). Note that

$\boldsymbol{f}(t)=\boldsymbol{f}_{*}(t)-\gamma[\boldsymbol{\sigma} \times(\boldsymbol{k} \times \boldsymbol{\sigma})-(\boldsymbol{\sigma}-\boldsymbol{k}) \log (\gamma \theta)]$,

which implies $\boldsymbol{f}(t)-\boldsymbol{f}\left(t_{0}\right)=\boldsymbol{f}_{*}(t)-\boldsymbol{f}_{*}\left(t_{0}\right)$ since the second term on the right-hand side of (39) is time-independent.

The solution (35)-(37) completely coincides with the solution for moving mass monopoles found by Kopeikin \& Schäfer (1999) and given in Appendix C in explicit form, provided that in the Kopeikin-Schäfer solution one assumes the body to move with a constant velocity. Note that if one computes also $\boldsymbol{k}=\boldsymbol{v} / \mathrm{c}$ and the corresponding quantities $\gamma$ and $\theta$ at the retarded moment $t_{*}$ the solution (37) for $\Delta v_{\mathrm{p}}$ becomes equivalent to the Kopeikin-Schäfer solution and is, therefore, correct for an arbitrarily moving mass monopole.

\section{Concluding remarks}

Using the well-known light propagation laws in the Schwarzschild field in the first post-Minkowskian approximation and the Lorentz transformation we have derived the postMinkowskian light propagation laws in the gravitational field of a uniformly moving mass monopole. In a similar way one can combine the Lorentz transformation and the light propagation laws in the gravitational field of a body with arbitrary multipole structure discussed by Kopeikin (1997) to derive the light propagation laws in the gravitational field of a uniformly moving body with full multipole structure. This will be done in a further publication.

\section{Appendix A: Lorentz transformation of some quantities}

Applying Lorentz transformations (3)-(6) to the quantities appearing in (26)-(30) one has

$$
\begin{aligned}
& \boldsymbol{\Sigma}=\frac{1}{\gamma \theta}\left(\boldsymbol{\sigma}-\frac{\gamma}{1+\gamma}(1+\gamma \theta) \boldsymbol{k}\right), \\
& \theta=1-\boldsymbol{\sigma} \cdot \boldsymbol{k}, \\
& \overline{\boldsymbol{\Sigma}}=\gamma(\boldsymbol{\sigma}-\boldsymbol{k}), \quad \overline{\boldsymbol{\Sigma}}^{i}=\left(\Lambda_{a}^{i}-\sigma^{i} \Lambda_{a}^{0}\right) \Sigma^{a}, \\
& \boldsymbol{R}=\boldsymbol{r}+\frac{\gamma^{2}}{1+\gamma}(\boldsymbol{k} \cdot \boldsymbol{r}) \boldsymbol{k}, \\
& \boldsymbol{r}=\boldsymbol{x}_{\mathrm{p} 0}+c \boldsymbol{\sigma}\left(t-t_{0}\right)-\boldsymbol{x}_{A}(t), \\
& |\boldsymbol{R}|=\left(|\boldsymbol{r}|^{2}+\gamma^{2}(\boldsymbol{k} \cdot \boldsymbol{r})^{2}\right)^{1 / 2}, \\
& \overline{\boldsymbol{R}}=\boldsymbol{r}-\gamma^{2}(\boldsymbol{k} \cdot \boldsymbol{r})(\boldsymbol{\sigma}-\boldsymbol{k}), \quad \bar{R}^{i}=\left(\Lambda_{a}^{i}-\sigma^{i} \Lambda_{a}^{0}\right) R^{a}, \\
& \boldsymbol{\Sigma} \cdot \boldsymbol{R}=\frac{1}{\gamma \theta} \boldsymbol{\sigma} \cdot \boldsymbol{r}-\gamma \boldsymbol{k} \cdot \boldsymbol{r}, \\
& \overline{\boldsymbol{D}}=\boldsymbol{r}-\frac{1}{\theta}(\boldsymbol{\sigma} \cdot \boldsymbol{r})(\boldsymbol{\sigma}-\boldsymbol{k}), \quad \bar{D}^{i}=\left(\Lambda_{a}^{i}-\sigma^{i} \Lambda_{a}^{0}\right) D^{a} .
\end{aligned}
$$

Note that for the transformation of $\boldsymbol{R}=\boldsymbol{X}_{\mathrm{p}}(T)-\boldsymbol{X}_{A}$ the Lorentz transformation should be applied for the following two pairs of events: (1) $\left(t, x_{\mathrm{p}}^{i}(t)\right)$ and $\left(T, X_{\mathrm{p}}^{a}(T)\right)$, and (2) $\left(t, x_{A}^{i}(t)\right)$ and $\left(T^{\prime}, X_{A}^{a}\right)$. Since the position $\boldsymbol{X}_{A}$ of the body in the reference system $\left(T, X^{a}\right)$ is time-independent it is allowed to use any moment $T^{\prime}$ to represent its position $\left(T^{\prime}, X_{A}^{a}\right)$ relative to that reference system. The moment $T^{\prime}$ is chosen in such a way that the event $\left(t, x_{A}^{i}(t)\right)$ corresponding to $\left(T^{\prime}, X_{A}^{a}\right)$ has in the reference system $\left(t, x^{i}\right)$ the same time coordinate as the event $\left(t, x_{\mathrm{p}}^{i}(t)\right)$. Using this scheme one easily derives that $R^{a}=\bar{\Lambda}_{i}^{a} r^{i}$.

\section{Appendix B: Expressions through the position of the body in retarded moment of time}

The retarded moment of time $t_{*}$ is a function of $t$ and the positions of the photon $\boldsymbol{x}_{\mathrm{p}}$ and the body $\boldsymbol{x}_{A}$ and is related to these quantities through the null cone equation

$t_{*}+\frac{1}{c}\left|\boldsymbol{r}_{*}\right|=t$

with

$\boldsymbol{r}_{*}=\boldsymbol{x}_{\mathrm{p} 0}+c \boldsymbol{\sigma}\left(t-t_{0}\right)-\boldsymbol{x}_{A}\left(t_{*}\right)$. 
The unperturbed coordinates of the photon are sufficient in (B.2) since we work in the first post-Newtonian approximation. For the case of a body moving with a constant velocity an exact relation between $\boldsymbol{r}$ and $\boldsymbol{r}_{*}$ can be derived. Using (A.5), (B.2), (B.1), (7) and $\boldsymbol{x}_{A}(t)=\boldsymbol{x}_{A}\left(t_{*}\right)+\boldsymbol{v}_{A}\left(t-t_{*}\right)$ one gets (see Sect. 14.1 of Jackson 1974 for a geometric interpretation)

$\boldsymbol{r}=\boldsymbol{r}_{*}-\left|\boldsymbol{r}_{*}\right| \boldsymbol{k}$.

Using $\boldsymbol{r}_{*}$ instead of $\boldsymbol{r}$ in the equations given in Appendix A one gets

$|\boldsymbol{R}|=\gamma\left(\left|\boldsymbol{r}_{*}\right|-\boldsymbol{k} \cdot \boldsymbol{r}_{*}\right)$,

$|\boldsymbol{R}|-\Sigma \cdot \boldsymbol{R}=\frac{1}{\gamma \theta}\left(\left|\boldsymbol{r}_{*}\right|-\boldsymbol{\sigma} \cdot \boldsymbol{r}_{*}\right)$,

$\overline{\boldsymbol{D}}=\boldsymbol{d}_{*}-\boldsymbol{\sigma} \times(\boldsymbol{k} \times \boldsymbol{\sigma}) \theta^{-1}\left(\left|\boldsymbol{r}_{*}\right|-\boldsymbol{\sigma} \cdot \boldsymbol{r}_{*}\right)$,

where $\theta$ is defined by (A.2) and

$\boldsymbol{d}_{*}=\sigma \times\left(\boldsymbol{r}_{*} \times \sigma\right)$

\section{Appendix C: The Kopeikin-Schäfer solution}

An exact post-Minkowskian solution for the light propagation in the gravitational field of moving mass monopoles has been first derived by Kopeikin \& Schäfer (1999). The authors do not write, however, their solution in explicit form needed for the purposes of this paper. Taking Eqs. (10), (26), (30), (31), (34) and (35) from Kopeikin \& Schäfer (1999) one can write the Kopeikin-Schäfer solution in the following explicit from:

$$
\begin{aligned}
\Delta \boldsymbol{x}_{\mathrm{p}}(t)= & -\frac{2 G M}{c^{2}}\left(\boldsymbol{f}^{K S}(t)-\boldsymbol{f}^{K S}\left(t_{0}\right)+\boldsymbol{g}\left(t_{0}, t\right)\right)+O\left(G^{2}\right) \\
\boldsymbol{f}^{\mathrm{KS}}(t)= & \gamma_{*}\left(\theta_{*} \frac{\boldsymbol{d}_{*}}{p_{*}}-\left(\boldsymbol{\sigma}-\boldsymbol{k}_{*}\right) \log p_{*}\right) \\
\boldsymbol{g}\left(t_{0}, t\right)= & \int_{t_{0}}^{t} \frac{\gamma_{*}^{3}}{c\left(\left|\boldsymbol{r}_{*}\right|-\boldsymbol{k}_{*} \cdot \boldsymbol{r}_{*}\right)}\left[\left(\gamma_{*}^{-2}\left(\boldsymbol{\sigma} \cdot \boldsymbol{a}_{*}\right)-\theta_{*}\left(\boldsymbol{k}_{*} \cdot \boldsymbol{a}_{*}\right)\right) \boldsymbol{d}_{*}\right. \\
& \left.+p_{*} \log p_{*}\left(\left(\boldsymbol{k}_{*} \cdot \boldsymbol{a}_{*}\right)\left(\boldsymbol{\sigma}-\boldsymbol{k}_{*}\right)-\gamma_{*}^{-2} \boldsymbol{a}_{*}\right)\right] \mathrm{d} t \\
\frac{1}{c} \Delta \boldsymbol{v}_{\mathrm{p}}(t)= & -\frac{2 G M}{c^{2}} \frac{\gamma_{*} \theta_{*}}{\left|\boldsymbol{r}_{*}\right|-\boldsymbol{k}_{*} \cdot \boldsymbol{r}_{*}} \\
& \times\left(\theta_{*} \frac{\boldsymbol{d}_{*}}{p_{*}}+\left(2-\theta_{*}\right) \boldsymbol{\sigma}-2 \boldsymbol{k}_{*}\right)+O\left(G^{2}\right)
\end{aligned}
$$

$$
\begin{aligned}
& \boldsymbol{a}_{*}=\ddot{\boldsymbol{x}}_{A}\left(t_{*}\right), \\
& \gamma_{*}=\left(1-\left|\boldsymbol{k}_{*}\right|^{2}\right)^{-1 / 2}, \\
& \theta_{*}=1-\boldsymbol{\sigma} \cdot \boldsymbol{k}_{*}, \\
& \boldsymbol{k}_{*}=\boldsymbol{k}\left(t_{*}\right),
\end{aligned}
$$

where $p_{*}$ is defined by (38), $\boldsymbol{d}_{*}$ by (B.7) and $\boldsymbol{r}_{*}$ by (B.2). Kopeikin \& Schäfer (1999) showed that the integral $\boldsymbol{g}\left(t, t_{0}\right)$ can be rewritten as a function of the retarded times $t_{*}$ and $t_{0 *}$ corresponding to the moments $t$ and $t_{0}$, so that the null cone equation is not required while computing $\boldsymbol{g}\left(t, t_{0}\right)$. However, this is not important for the present paper and we prefer to retain $\boldsymbol{g}\left(t, t_{0}\right)$ in the form given by (C.3).

Note that the Kopeikin-Schäfer solution is valid for arbitrarily moving bodies and that the velocity $\boldsymbol{v}$ of the body and the vector $\boldsymbol{k}=\boldsymbol{v} / \boldsymbol{c}$ are not necessarily constants. The velocity $\boldsymbol{v}$ should be computed for the retarded moment $t_{*}$ in (C.6)-(C.8). The actual trajectory of the body should be used in (B.1) and (B.2) to compute $t_{*}$ and $\boldsymbol{r}_{*}$.

\section{References}

Bienaymé, O., \& Turon, C. (ed.) 2002, GAIA: A European Space Project (Les Ulis: EDP Sciences)

ESA. 2000, GAIA: Composition, Formation and Evolution of the Galaxy, Concept and Technology Study Report (ESA-SCI[2000]4) (Noordwijk: ESA)

Hellings, R. W. 1986, AJ, 91, 650

Jackson, J. D. 1974, Classical Electrodynamics (New York: Wiley)

Klioner, S. A. 1989, Soobschch. Inst. Prik. Astron. No. 6 (Communications of the Institute of Applied Astronomy, No. 6, St. Petersburg, in Russian)

Klioner, S. A. 1991, in Geodetic VLBI: Monitoring Global Change, ed. W. E. Carter, NOAA Technical Report NOS 137 NGS 49, 188 (Washington D.C.: American Geophysical Union)

Klioner, S. A. 2003, AJ, 125, 1580

Klioner, S. A., \& Peip, M. 2003, Numerical Simulations of the Light Propagation in Gravitational Field of Moving Bodies, A\&A, submitted

Klioner, S. A., \& Kopeikin, S. M. 1992, AJ, 104, 897

Kopeikin, S. M. 1997, J. Math. Phys., 38, 2587

Kopeikin, S. M., \& Schäfer, G. 1999, Phys. Rev. D, 60, No. 124002

Kopeikin, S. M., \& Mashhoon, B. 2002, Phys. Rev. D, 65, No. 64025

Perryman, M. A. C., et al. 2001, A\&A, 369, 339

Shao, M. 1998, Proc. SPIE, 3350, 536

Will, C. M. 1993, Theory and experiment in gravitational physics (Cambridge: Cambridge University Press) 